\title{
Niveles de asimilación económica del estado de Guerrero
}

Levels of economic assimilation of the state of Guerrero

Níveis de assimilação econômica do estado de Guerrero

\author{
Neftalí García Castro \\ Universidad Autónoma de Guerrero, México \\ ngarciaca@conacyt.mx
}

Salvador Villerías Salinas

Universidad Autónoma de Guerrero, México

svilleriass@gmail.com

Pedro Vidal Tello Almaguer

Universidad Autónoma de Guerrero, México

pvidalt@hotmail.com

\section{Resumen}

Este trabajo tiene como objetivo central exponer la configuración del estado de Guerrero, con base en la teoría de la asimilación económica y la tipificación probabilística. Esta propuesta teórico-metodológica de la geografía económica coadyuva a la interpretación de la relación que guardan los componentes sociales, económicos y físicos, en un espacio particular. La metodología empleada es fundamental para la construcción de tipologías que compendian el comportamiento de los indicadores seleccionados, con la finalidad de ponderar desproporciones territoriales, las cuales son interpretadas a la luz de los escenarios socio-territoriales reconocidos por los postulados de la asimilación económica. Este artículo revela once niveles que dan cuenta de un proceso de asimilación económica significativo en aquellas demarcaciones que contienen centros turísticos consolidados (Acapulco e Ixtapa-Zihuatanejo), función política-administrativa (Chilpancingo), un papel notable en el abastecimiento regional de bienes y servicios o aquellos que reportan actividades agropecuarias con rendimientos considerables.

Palabras clave: asimilación económica, tipología, territorio, Guerrero, México. 


\section{Abstract}

This work aims to expose the configuration of the State of Guerrero, based on the theory of economic assimilation and the probabilistic classification. This theoretical-methodological proposal of economic geography contributes to the interpretation of the relationship that are social, physical, and economic components in a particular space. The methodology used is fundamental for the construction of typologies that summarize the behavior of the selected indicators, in order to consider territorial imbalances, which are interpreted in the light of socioterritorial scenarios recognized by the postulates of economic assimilation. This article reveals eleven levels that account for a significant economic assimilation process in those districts that contain consolidated resorts (Acapulco and Ixtapa-Zihuatanejo), political and administrative function (Chilpancingo), a notable role in the regional supply of goods and services, or those that report agricultural activities with substantial yields.

.Key Words: economic assimilation, typology, territory, Guerrero, México.

\section{Resumo}

Este trabalho tem como objetivo expor o estado de configuração de Guerrero, com base na teoria de assimilação econômica e classificação probabilística. Esta proposta teórica e metodológica da geografia econômica contribui para a interpretação da relação a componentes sociais, econômicos e físicos em um espaço particular. A metodologia é fundamental para a construção de tipologias que resumem o comportamento dos indicadores selecionados, a fim de ponderar desproporções territoriais, que são interpretadas à luz dos cenários sócio-territorial, reconhecidos pelos postulados de assimilação econômica. Este artigo revela os níveis de onze que respondem por um processo de assimilação económica significativa nesses distritos que contêm consolidadas resorts (Acapulco e Ixtapa-Zihuatanejo), função político-administrativa (Chilpancingo), um papel notável na oferta regional de bens e serviços ou aqueles que relataram atividades agrícolas com rendimentos consideráveis.

Palavras-chave: assimilação econômica, tipologia, território, Guerrero, México.

Fecha recepción: Enero 2016

Fecha aceptación: Junio 2016 


\section{Introducción}

La evolución geo-histórica acaecida sobre un entorno físico variado, ha dado un carácter especial y propio a Guerrero. Así lo permiten vislumbrar los contrastes socioeconómicos que prevalecen entre sus distintos municipios. De la región Norte a la Costa y de la Montaña a la Tierra Caliente, la entidad es biodiversa y cuenta con un vasto patrimonio histórico-etnográfico-cultural que le confiere un lugar significativo dentro de la vida nacional. Sin embargo, sólo unos cuantos sitios, entre ellos Acapulco, Ixtapa-Zihuatanejo, Chilpancingo, Iguala y Taxco, detentan la mayor concentración de habitantes, inversiones económicas y actividades productivas rentables, sobre todo pertenecientes al sector terciario. Asimismo, estas demarcaciones albergan los núcleos urbanos más importantes de la entidad. En la mayoría de ellos, la expansión económicodemográfica reciente se debe a su actividad turística, su función político-administrativa o bien su papel notable en el abastecimiento regional de bienes y servicios.

La presente investigación examina las particularidades económico-territoriales del estado de Guerrero, desde la óptica de la asimilación económica y la tipificación probabilística. Ambos referentes cognoscitivos son básicos para el análisis integral de las características socioterritoriales de una entidad como Guerrero, ya que el enfoque sistémico de la asimilación, así como la confección de tipología y cartografía temática, es un aporte substancial a la elucidación de las disparidades regionales de esta entidad mexicana. El trabajo está estructurado de la manera siguiente: en el primer apartado se exponen las principales características socioeconómicas que distinguen al estado de Guerrero. En las secciones subsecuentes se acotan los antecedentes cognoscitivos de esta investigación, se explican las particularidades metodológicas de la tipificación probabilística y se revelan los niveles de asimilación económica hallados en los municipios guerrerenses.

\section{Características socioeconómicas de Guerrero}

Guerrero se localiza al sur de la república mexicana y tiene una superficie de $63794 \mathrm{~km}^{2}$ (3.2\% del territorio nacional). La entidad se divide en las regiones siguientes: Norte, Centro, Acapulco, Tierra Caliente, Montaña, Costa Grande y Costa Chica. La Montaña concentra el mayor número de municipios, diecinueve en total (figura 1) (Gobierno del estado de Guerrero, 2016). Junto con la Costa Chica constituyen las áreas con presencia significativa de población indígena. En 2010, 
ésta sumó 475099 personas, $7 \%$ del total nacional. Asimismo, $36 \%$ eran nahuas, $29 \%$ mixtecos, $25 \%$ tlapanecos y $9 \%$ amuzgos (INEGI, 2016).

Figura 1. Guerrero: división político-administrativa, 2010

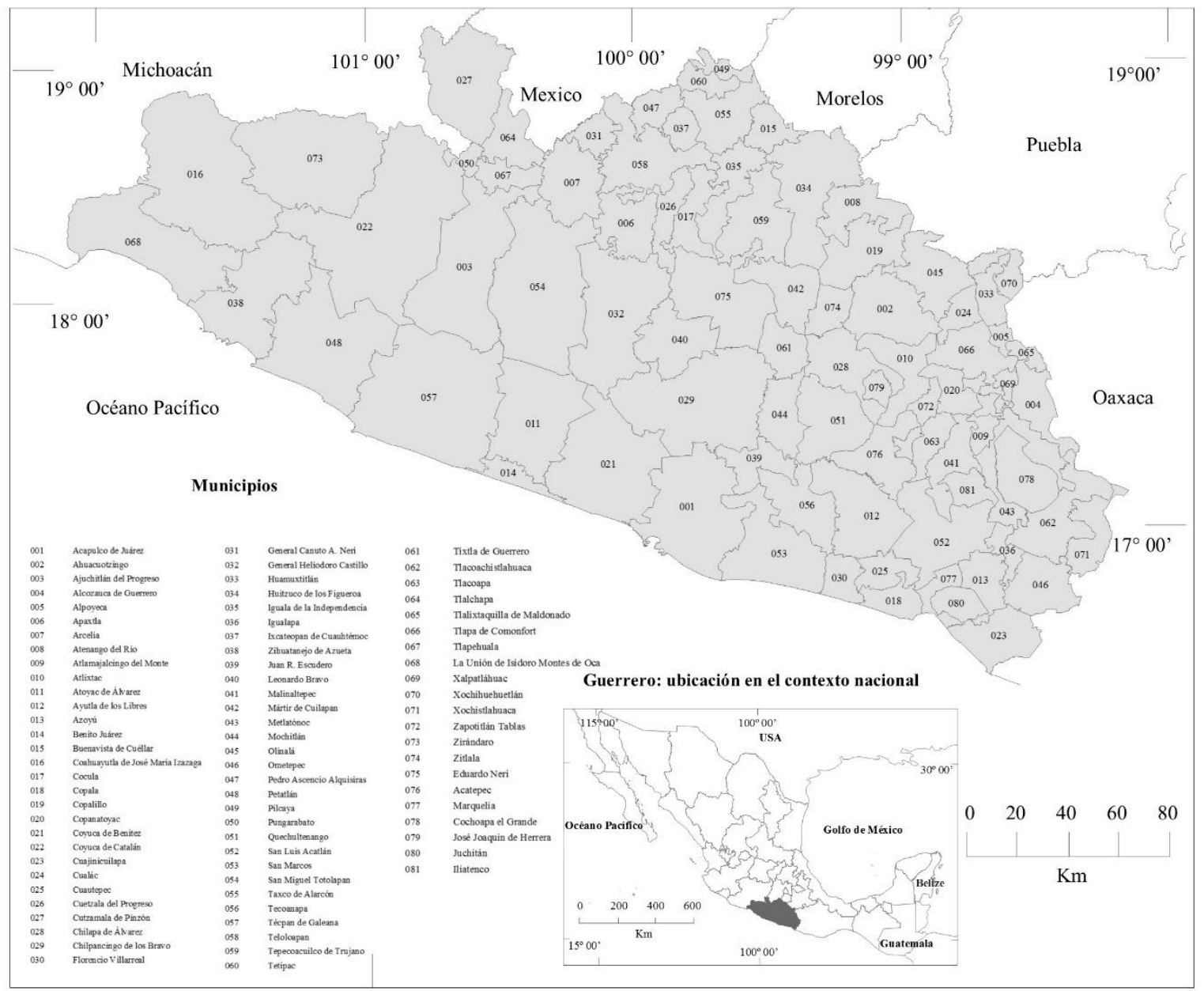

Fuente: elaborado con base en INEGI, 2016.

El estado de Guerrero tiene 3388768 habitantes (3\% de la población mexicana). Del total de guerrerenses, $23 \%$ reside en Acapulco (789 971 habitantes), $7 \%$ en Chilpancingo (241 717 habitantes), $4.1 \%$ en Iguala (140 363 habitantes), $3.6 \%$ en Chilapa (120 790 habitantes), $3.5 \%$ en Zihuatanejo de Azueta (118 211 habitantes), $3.1 \%$ en Taxco (104 053 habitantes), $2.4 \%$ en Tlapa (81 419 habitantes) y $2.2 \%$ en Coyuca de Benítez (73 460 habitantes). Esos ocho 
municipios concentran $49 \%$ de la población estatal. El resto se distribuye en las otras 73 circunscripciones, de las cuales 54 no rebasan individualmente al $1 \%$ del total de guerrerenses (INEGI, 2016).

Sólo unos cuantos municipios han recibido impulso federal, estatal y del capital privado, relacionado con actividades como el turismo, la minería o las agroindustrias, entre ellos Acapulco, Iguala, Zihuatanejo de Azueta, Taxco y Eduardo Neri. Asimismo, hay pocos espacios que deben su relevancia territorial a la función político-administrativa (Chilpancingo), además han desempeñado un papel fundamental en el abastecimiento regional de bienes y servicios (Tixtla, Tlapa, Arcelia, Teloloapan, Atoyac de Álvarez, Petatlán) (García, 2011).

El estado ha mostrado un constante éxodo de campesinos que buscan mejores ingresos en las principales ciudades de Guerrero, en otras entidades federativas o en Estados Unidos. Cada año, aproximadamente, 73000 guerrerenses se desplazan a Oregón, California, Arizona, Mississippi, Florida, Nueva York, Virginia o Carolina del Norte. También, cada verano cerca de 128000 jornaleros del estado emigran para laborar en los campos agrícolas de Sonora, Chihuahua, Baja California o Sinaloa. En años recientes, el territorio guerrerense se ha posicionado entre las cinco entidades de la república mexicana que presentan mayor emigración (INEGI, 2016).

De acuerdo con el Consejo Nacional de Evaluación de la Política de Desarrollo Social (CONEVAL), en Guerrero hay 1112000 personas en situación de pobreza extrema, esto es 31.7 $\%$ de los guerrerenses. En la entidad, $78.5 \%$ de los habitantes tiene carencias vinculadas con el acceso a la seguridad social; $59 \%$ asociadas con los servicios básicos en sus viviendas; $39.4 \%$ ligadas con el acceso a la alimentación; $33.4 \%$ relacionadas con la calidad y dimensiones de la vivienda; $26.8 \%$ presenta rezago educativo y $25.4 \%$ muestra deficiencias en el acceso a los servicios de salud (CONEVAL, 2016). 


\section{Sustento cognoscitivo}

En la literatura especializada se señala que la asimilación económica debe ser concebida como la gradual incorporación de distintos espacios a la vida económica del territorio al que pertenecen. Las particularidades de esa integración son resultado del momento en que tiene lugar, las relaciones sociales en las que se inscribe y las características físicas del espacio en cuestión. En relación con estos factores, es importante especificar las cuestiones siguientes: primero, con frecuencia acontecen simultáneos procesos de asimilación económica en un territorio, situación asociada con los diferentes estadios de desarrollo de las fuerzas productivas inherentes a él. Segundo, también suele presentarse el abandono de algunos sitios ya asimilados; debido, generalmente, al agotamiento, destrucción o pérdida del valor de sus recursos naturales (García, 1993).

La teoría de la asimilación económica tiene como objetivo fundamental revelar el grado de dominio que se tiene sobre un territorio mediante la evaluación de la intensidad de utilización de éste, el nivel de desarrollo de sus fuerzas productivas, la historia de poblamiento y otras particularidades del territorio abordado (Zailsev, 1972 citado por García, 1993). El empleo de este cuerpo teórico es útil para proponer criterios de ordenamiento; al revelar, conjuntamente, la celeridad con la que los territorios analizados se integran a ciertos procesos socioeconómicos, las limitantes que aún constituyen ciertos atributos del entorno físico-geográfico y los nexos entre la intensidad del uso económico que manifiesta un espacio concreto y su problemática ambiental. Consecuentemente se puede:

Establecer proyecciones territoriales a partir de los patrones que se detecten y, sobre todo, incidir mediante el conocimiento más profundo de los procesos territoriales en una mejor organización para el aprovechamiento del espacio, una más eficiente utilización de los recursos, una más regional distribución de las fuerzas productivas y una mejor relación con la naturaleza en el país (Propin, 1989 citado por García, 1993, p. 73).

La teoría está integrada por tres líneas cognoscitivas básicas que son: los niveles, los tipos y los grados de asimilación económica de un territorio (figura 2). La primera ha sido empleada en un número significativo de investigaciones, para evaluar las disparidades socioeconómicas que existen al interior del espacio abordado. En esas obras se define al nivel como la expresión medible del proceso de interacción de factores múltiples como los económicos, políticos, 
culturales y físico-geográficos que causan la "situación geográfica" de un territorio concreto (Privalovskaya, 1982 citada por Propin, 2003, p. 60). Tal dimensión cualitativo-cuantitativa es determinada mediante el tratamiento metodológico de criterios previamente seleccionados y relacionados con determinadas unidades político-administrativas. El resultado es una imagen integral de la presencia heterogénea de la población y sus actividades productivas. Por lo tanto, los distintos niveles de asimilación económica "no deben ser asociados con supuestos patrones de desarrollo económico o social" (Propin y Sánchez, 1998, p. 60).

Figura 2. Categorías investigativas de la asimilación económica del territorio

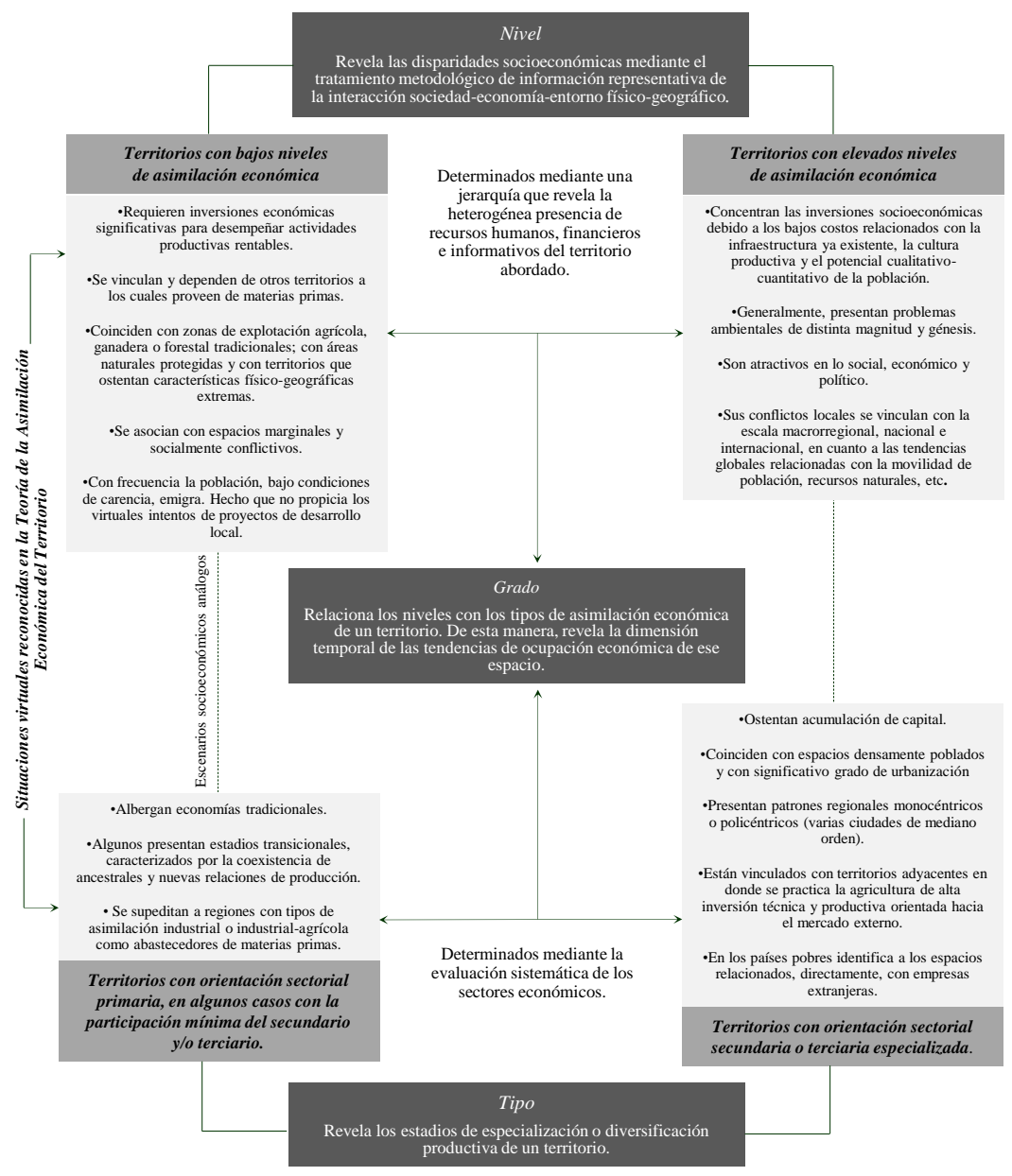

Fuente: elaborado con base en García, 2011. 


\section{Método}

En este trabajo se emplea información generada por el Instituto Nacional de Estadística y Geografía (INEGI). Las investigaciones precedentes, sustentadas en la asimilación económica, han utilizado las variables siguientes: extensión territorial, número de habitantes, población urbana, valor anual de la producción agrícola e industrial y longitud de las vías de comunicación terrestre. A partir de esas cinco variables se generaron indicadores socioeconómicos que permiten vislumbrar las disparidades espaciales que imperan en un territorio. A continuación se presentan sus principales características.

Densidad de población (DP). Muestra la relación entre el total de habitantes de una unidad espacial de análisis y el área de ésta.

Grado de urbanización (GU). Es un valor porcentual que evidencia la proporción de población que reside en un asentamiento urbano, sitios en los que viven 15000 o más personas (Unikel, 1978), respecto al número total de habitantes que registra la unidad espacial analizada.

Concentración regional de las tierras con riego (CTA). El valor correspondiente a este indicador revela el porcentaje de tierras con regadío, presentes en cada municipio, en relación a la superficie agrícola estatal de ese tipo.

Concentración espacial de la industria (CTI). Alude a la cifra que resulta al dividir el valor de la producción de las ramas básicas del sector secundario entre la superficie de la unidad espacial en la que se desarrollan tales actividades económicas.

Densidad vial (DV). Así se denominó a la relación que existe entre la longitud de la red vial que ostenta cada unidad espacial de análisis y el área de la misma. En trabajos subsecuentes también se pondera la amplitud de las vías ferroviarias, debido a su trascendencia en la dinámica económica del territorio evaluado.

El manejo estadístico de estos indicadores está regulado por el método de tipificación probabilística cuyas particularidades están expuestas en los de Propin (2003), Hernández (2007), Mollinedo (2008), Ortiz, Villaseñor y Gerónimo (2009), Aguilar (2013) y Villagrán (2013). A continuación se sintetizan los pasos que se siguieron para determinar los niveles de asimilación económica del estado de Guerrero. 
a. Conformación de las matrices de datos. La información estadística se integró en dos cuadros que muestran el comportamiento cuantitativo de las variables e indicadores, en cada uno de los ochenta y un municipios. Este compendio de datos es la plataforma básica de trabajo para determinar los niveles de asimilación económica de un territorio (tabla 1 y 2).

Tabla 1. Ejemplo del comportamiento cuantitativo de las variables socioeconómicas

\begin{tabular}{ccccccc} 
Municipio & $\begin{array}{c}\text { Extensión } \\
\text { territorial km }\end{array}$ & $\begin{array}{c}\text { Población } \\
\text { Total }\end{array}$ & $\begin{array}{c}\text { Población } \\
\text { urbana }\end{array}$ & $\begin{array}{c}\text { Superficie } \\
\text { irrigada ha }\end{array}$ & $\begin{array}{c}\text { Producción } \\
\text { industrial usD }\end{array}$ & $\begin{array}{c}\text { Longitud } \\
\text { vial } \mathrm{km}\end{array}$ \\
\hline Acapulco de Juárez & 1882.6 & 789971 & 673479 & 1558 & 476290278 & 548.5 \\
Ahuacuotzingo & 388.4 & 25027 & 0 & 118.5 & 114862 & 395.5 \\
Ajuchitlán del Progreso & 1983.6 & 38203 & 0 & 6563.75 & 988746 & 388.0 \\
Alcozauca de Guerrero & 551.6 & 18971 & 0 & 123.25 & 152545 & 211.2 \\
Alpoyeca & 155.4 & 6637 & 0 & 938.25 & 225804 & 13.0 \\
\hline
\end{tabular}

Fuente: elaborado con base en INEGI, 2016.

Tabla 2. Ejemplo del comportamiento cuantitativo de los indicadores socioeconómicos

\begin{tabular}{cccccc} 
Municipio & $\begin{array}{c}\text { DP } \\
\text { hab/km² }\end{array}$ & $\begin{array}{c}\text { GU } \\
\text { CTA }\end{array}$ & $\begin{array}{c}\text { CPI } \\
\text { USD/km }\end{array}$ & $\begin{array}{c}\text { DV } \\
\mathbf{k m} / \mathbf{k m}^{2}\end{array}$ \\
Acapulco de Juárez & 419.6 & 85.3 & 1.6 & 252996.0 & 0.3 \\
Ahuacuotzingo & 64.4 & 0 & 0.1 & 295.7 & 1.0 \\
Ajuchitlán del Progreso & 19.3 & 0 & 6.6 & 498.5 & 0.2 \\
Alcozauca de Guerrero & 34.4 & 0 & 0.1 & 276.5 & 0.4 \\
Alpoyeca & 42.7 & 0 & 0.9 & 1453.1 & 0.1 \\
\hline
\end{tabular}

Fuente: elaborado con base en la tabla 1.

b. Clasificación cualitativa de los indicadores. Los valores de cada uno de los indicadores se ordenaron de manera descendente para observar su variación. Esto facilitó la conformación de cinco rangos como vías de generalización cualitativa (Propin, 2003). Los calificativos que se emplean y su codificación numérica son: muy alta (5), alta (4), media (3), baja (2) y muy baja (1) (tabla 3). Sucesivamente, se elaboró una nueva matriz donde se sustituyeron los montos de los cinco indicadores socioeconómicos por combinaciones 
de índices clasificatorios que corresponden a cada municipio, según el rango asignado a su valor (tabla 4).

Tabla 3. Clasificación cualitativa de indicadores

\begin{tabular}{cccccc}
$\begin{array}{c}\text { Indicador } \\
\text { Rango }\end{array}$ & $\begin{array}{c}\mathrm{DP} \\
\mathbf{h a b} / \mathbf{k m}^{2}\end{array}$ & $\begin{array}{c}\mathrm{GU} \\
\%\end{array}$ & $\begin{array}{c}\mathrm{CTA} \\
\%\end{array}$ & $\begin{array}{c}\mathrm{CPI} \\
\text { miles USD } / \mathbf{k m}^{2}\end{array}$ & $\begin{array}{c}\mathrm{DV} \\
\mathbf{k m} / \mathbf{k m}^{2}\end{array}$ \\
Muy alto & $>300$ & $>80$ & $>6.0$ & $>260$ & $>1.0$ \\
Alto & $>200, \ldots, 300$ & $>60, \ldots, 80$ & $>3.96, \ldots, 6.0$ & $>70, \ldots, 260$ & $>0.6, \ldots, 1.0$ \\
Medio & $>50, \ldots, 200$ & $>50, \ldots 60$ & $>2.64, \ldots, 3.96$ & $>14.5, \ldots, 70$ & $>0.4, \ldots, 0.6$ \\
Bajo & $>35, \ldots, 50$ & $20, \ldots, 50$ & $>1.32, \ldots, 2.64$ & $>3.5, \ldots, 14.5$ & $>0.2, \ldots, 0.4$ \\
Muy bajo & $<35$ & 0 & $<1.32$ & $<3.5$ & $<0.2$ \\
\hline
\end{tabular}

Fuente: elaborado con base en el comportamiento de los indicadores ponderados.

Tabla 4. Ejemplos de indicadores ponderados

\begin{tabular}{lccccc}
\multicolumn{1}{c}{ Municipio } & DP & GU & CTA & CPI & DV \\
Acapulco de Juárez & 5 & 5 & 2 & 4 & 2 \\
Acatepec & 3 & 1 & 1 & 1 & 2 \\
Ahuacuotzingo & 3 & 1 & 1 & 1 & 5 \\
Ajuchitlán del Progreso & 1 & 1 & 5 & 1 & 1 \\
Alcozauca de Guerrero & 1 & 1 & 1 & 1 & 2 \\
Alpoyeca & 2 & 1 & 1 & 1 & 1 \\
\hline
\end{tabular}

Fuente: elaborado con base en las tablas 2 y 3.

c. Conformación de las nubes tipológicas. Fue indispensable elaborar una lista de las combinaciones que se presentaron, así como de la frecuencia de cada una de ellas (tabla 5). Eso permitió conocer todos los códigos que mostró la entidad, los más recurrentes fueron considerados núcleos a partir de los cuales se ligaron otros que indican la existencia de circunscripciones con comportamiento socioeconómico análogo. Se usó línea continua cuando los códigos se desviaron entre sí en el rango de un solo indicador. Las rayas segmentadas conectaron aquellos que no cumplieron con la condición anterior. En ese caso, fue esencial ponderar la afinidad que hay entre una determinada nube y el código que se anexa. 
Tabla 5. Frecuencia de códigos

\begin{tabular}{lcccccccc} 
Código & Casos & Código & Casos & Código & Casos & Código & Casos \\
11111 & 9 & 21111 & 1 & 23523 & 1 & 32322 & 1 \\
11112 & 12 & 21112 & 8 & 31112 & 5 & 32414 & 1 \\
11113 & 1 & 21113 & 3 & 31114 & 1 & 33122 & 1 \\
11114 & 1 & 21114 & 1 & 31115 & 1 & 33131 & 1 \\
11212 & 3 & 21121 & 1 & 31122 & 1 & 33251 & 1 \\
11213 & 1 & 21212 & 1 & 31123 & 2 & 34131 & 2 \\
11311 & 2 & 21312 & 1 & 31125 & 1 & 42124 & 1 \\
11412 & 1 & 21314 & 1 & 31213 & 1 & 43144 & 1 \\
11511 & 1 & 22112 & 2 & 31232 & 1 & 45141 & 1 \\
12411 & 1 & 22322 & 1 & 31312 & 1 & 55242 & 1 \\
12522 & 1 & 23131 & 1 & 31421 & 1 & & \\
\hline
\end{tabular}

Fuente: elaborado con base en el comportamiento de los indicadores ponderados

Posteriormente, fue necesario establecer el nivel de asimilación económica que refiere cada grupo de códigos conformado. Éstos se jerarquizaron con base en el comportamiento de los coeficientes de correlación exhibidos entre los cinco indicadores socioeconómicos. Los que ostentan una reciprocidad alta se tomaron en cuenta para decidir qué lugar ocuparía una determinada nube tipológica (Propin, 2003). En esta ocasión hubo valores significativos entre la concentración espacial de la producción industrial y el grado de urbanización, así como entre la primera y la densidad de población (tabla 6). A través del procedimiento referido se revelaron los grupos de municipios con cualidades básicas similares que integran los distintos niveles (figura 2).

Tabla 6. Coeficientes de correlación entre indicadores

\begin{tabular}{|c|c|c|c|c|c|}
\hline Indicadores & DP & & & & \\
\hline DP & & GU & & & \\
\hline GU & 0.541530165 & & CTA & & \\
\hline CTA & -0.05735876 & 0.103817078 & - & CPI & \\
\hline CPI & 0.620795781 & 0.805706316 & 0.049274499 & - & CV \\
\hline CV & 0.304215233 & -0.13816334 & -0.036182488 & -0.089078686 & \\
\hline
\end{tabular}

Fuente: elaborado con base en el comportamiento de los indicadores ponderados. 
Figura 2. Agrupación de los códigos en nubes tipológicas

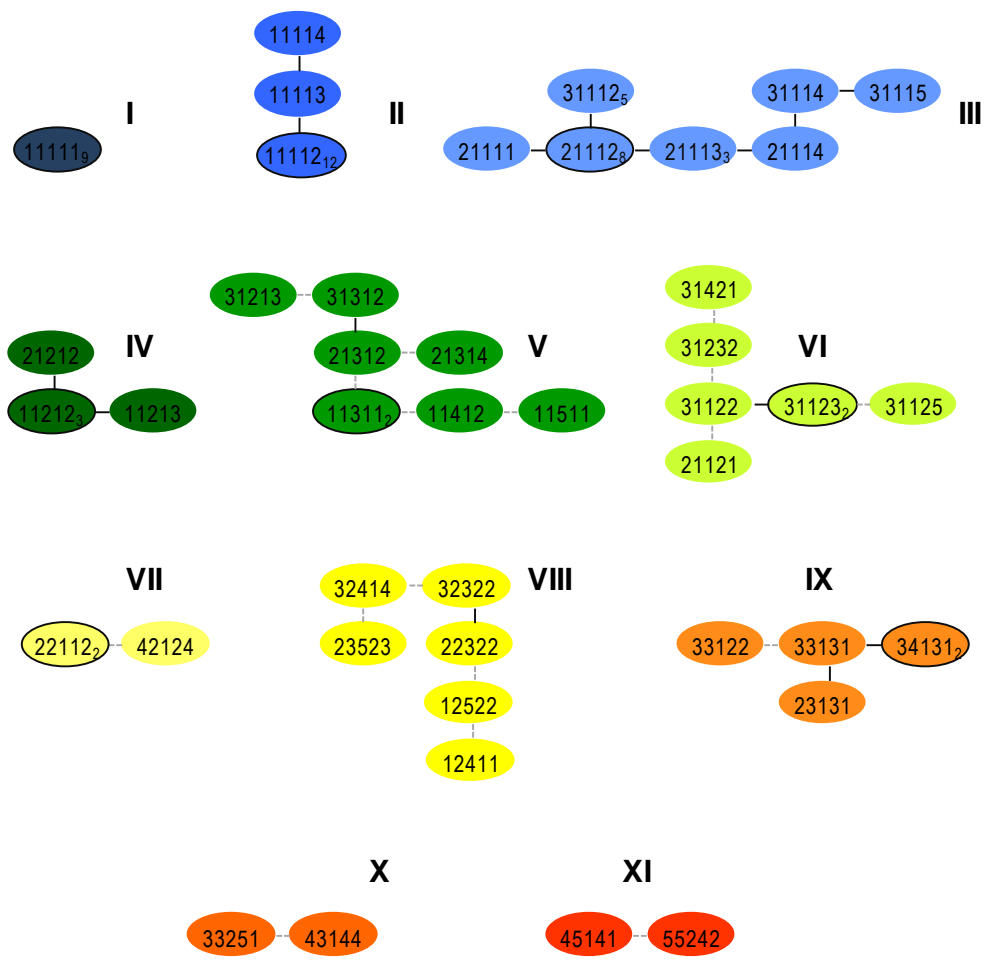

Fuente: elaborado con base en el comportamiento de los indicadores ponderados.

d. Revelación de la tipología. Cada nube conformada se codificó mediante una nomenclatura que tomó en cuenta el número de ocasiones en las que se presentó el rango correspondiente a cada indicador socioeconómico (tabla 7). Las variaciones de éste se expresaron de las cuatro formas ejemplificadas a continuación (Propin, 2003).

1: Señaló la existencia de valores muy bajos en más de $90 \%$ de los códigos de una nube.

23: Indicó el predominio de un indicador con rango bajo (entre 80 y $90 \%$ del conjunto de circunscripciones que conforman al nivel). Los casos secundarios se presentaron como subíndices.

2(3): Reveló el predomino relativo de valores bajos (entre 50 y menos de $80 \%$ de los municipios muestra esa condición). Se emplearon subíndices y paréntesis para expresar otros rangos que ostentó el indicador en cuestión.

2,3: Significó que los montos bajos y medios aparecieron con la misma frecuencia. Esa situación equilibrada se registró mediante el empleo de números de igual dimensión. 
Tabla 7. Características de los indicadores por niveles

\begin{tabular}{|c|c|c|c|c|c|}
\hline $\begin{array}{l}\text { Indicadores } \\
\text { Niveles }\end{array}$ & DP & GU & CTA & CPI & DV \\
\hline I & 1 & 1 & 1 & 1 & 1 \\
\hline II & 1 & 1 & 1 & 1 & 234 \\
\hline III & $2(3)$ & 1 & 1 & 1 & $2_{(3)}$ \\
\hline IV & 12 & 1 & 2 & 1 & $2_{3}$ \\
\hline V & $1_{\text {(2) (3) }}$ & 1 & (2) 3 (4) (5) & 1 & $1,2_{\text {(3) (4) }}$ \\
\hline VI & 32 & 1 & $1_{(2)(4)}$ & 23 & $1,2,3_{(5)}$ \\
\hline VII & $2(4)$ & 2 & 1 & $1_{(2)}$ & $2(4)$ \\
\hline VIII & $1,2,3$ & 23 & $3,4,5$ & $2(1)$ & (1) 2 (3) (4) \\
\hline IX & 23 & 3 (4) & 1 & 23 & 12 \\
\hline $\mathbf{X}$ & 34 & 3 & 12 & 45 & 14 \\
\hline XI & 45 & 5 & 12 & 4 & 12 \\
\hline
\end{tabular}

Fuente: elaborado con base en el comportamiento de los indicadores ponderados.

\section{Resultados}

En el estado de Guerrero se descubrieron once niveles y el predominio de indicadores con poca trascendencia cuantitativa reveló muy baja asimilación económica en cuarenta y tres municipios (figura 3). Un panorama socioeconómico análogo tuvo lugar en todas las demarcaciones con nivel IV, V y VI. Éstos advirtieron la existencia de espacios con baja asimilación (en total veinte jurisdicciones), pues, aunque en proporción menor que en los tres primeros estratos, fueron recurrentes los valores bajos y muy bajos. Sólo algunas circunscripciones reportaron cierta importancia en los montos de población relativa, concentración regional de tierras con riego o de la producción industrial.

Con base en lo descrito, se halló que $77 \%$ de los municipios guerrerenses manifestaron asimilación económica mínima. La mayoría de ellos tiene en común las características siguientes: alberga asentamientos humanos pequeños, dispersos y escasamente articulados por algún tipo de carretera. Posee niveles altos de pobreza, grados elevados de marginación, cuenta con cantidad substancial de familias campesinas. Por lo general, éstas no captan inversiones económicas que den pauta para desempeñar actividades agropecuarias o silvícolas rentables y sustentables. Además, el sector secundario tampoco ha sido estimulado significativamente por ninguno de los 
tres niveles de gobierno o la iniciativa privada, las inversiones registradas son escasas (García, 2011).

\section{Figura 3. Guerrero: niveles de asimilación económica}

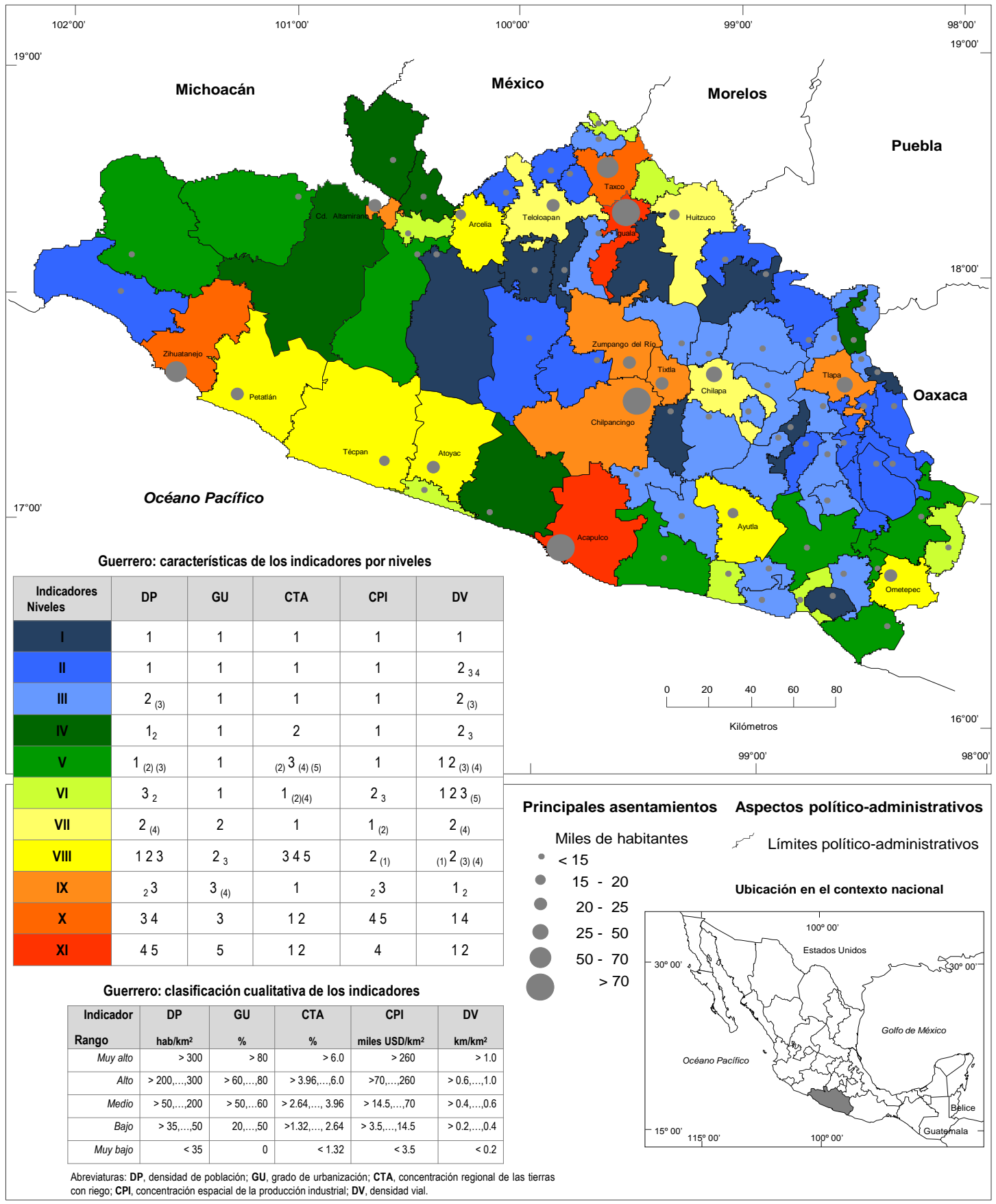

Fuente: elaborado con base en el comportamiento de los indicadores ponderados. 
Las categorías VII y VIII están asociadas a una condición de asimilación económica intermedia, la cual se identificó en nueve municipios. Éstos se ubican en el área septentrional de Guerrero, a lo largo de la costa y en menor medida en la región Centro. Un rasgo sobresaliente fue que sus cabeceras municipales tienen más de 15000 habitantes y fungen como centros de abasto comercial para las circunscripciones adyacentes. Asimismo, la agricultura y la ganadería poseen cierta relevancia económica debido a que hay algunas áreas con características físicas favorables para el desempeño de esas actividades o bien se les ha dotado de infraestructura hidroagrícola, desde hace varios decenios. Otro rasgo característico es la sobreexplotación de los recursos forestales, situados en las partes serranas de las demarcaciones costeras con asimilación económica media.

En tanto, los espacios altamente asimilados son aquellos que exhibieron nivel IX y X. En tal circunstancia se encontraron siete municipios que presentan la dinámica socioeconómica señalada a continuación. Tienen una cantidad relevante de habitantes del estado y cuentan con ciudades con más de 20000 personas. Por lo general, esos asentamientos asumen un papel notable en el abastecimiento regional de bienes y servicios; han recibido, reiteradamente, en menor o mayor proporción inversiones económicas y se conciben como lugares atractivos para el resto de los guerrerenses, quienes migran a esos sitios y debido a su escasa calificación laboral suelen desempeñar labores relacionadas con el comercio o los servicios. Lógicamente, esa situación ha originado expansión demográfica y crecimiento del sector terciario en esas localidades de la entidad. Las características puntualizadas en este párrafo se acentúan en los municipios con la mayor asimilación económica de Guerrero, descubierta en Acapulco e Iguala (nivel XI).

\section{Discusión}

La ponderación del proceso de asimilación económica en Guerrero podría ser un referente inicial para el diseño de las políticas públicas que tengan como objetivo fundamental incentivar la transformación positiva del panorama económico-productivo estatal. La determinación de los niveles de asimilación económica agiliza la identificación de las áreas donde es factible impulsar proyectos económicos concretos y, por otro lado, aquellas que demandan el rediseño de los instrumentos utilizados por la política económica y social estatal para elevar la competitividad de las diferentes circunscripciones. De acuerdo con los niveles identificados en el territorio 
guerrerense, este tipo de acciones apremian, pues se halló que $77 \%$ de los municipios manifestaron asimilación económica mínima.

La tipología revelada, como suele ocurrir en las investigaciones de corte geográfico-estadístico que recurren a los municipios como unidad de análisis, brinda una imagen general de la cobertura espacial de las características socioeconómicas que expresa cada nivel de asimilación económica. En ese sentido, el contenido de la cartografía presentada resume los patrones territoriales derivados de la ponderación de la categoría abordada en esta investigación; no obstante, un diagnóstico más preciso de la configuración económico-productiva municipal requiere del análisis de las características físicas y la conformación histórica estatal, así como el escenario social contemporáneo. Así, como apuntan los especialistas en esta vertiente de la Geografía Económica, resulta crucial afinar los escenarios definidos por la tipificación probabilística mediante la consulta de un cúmulo de fuentes complementarias de información de suma relevancia por su tratamiento de la realidad espacial, aunque limitadas en cuanto a su carácter cuantitativo.

\section{Conclusiones}

El conjunto de elementos cognoscitivos revelado en esta investigación sobre el estado de Guerrero, derivado de la aplicación de la teoría de la asimilación económica del territorio, constituye una plataforma cognoscitiva cualitativo-cuantitativa útil para la dilucidación de los desequilibrios regionales que caracterizan a este espacio de la república mexicana desde hace siglos. Sin embargo, cabe destacar que es a partir de la segunda mitad del siglo XX cuando se agudizan las disparidades territoriales por la consolidación de Acapulco e Ixtapa-Zihuatanejo como importantes centros turísticos ya que, para lograrlo, los gobiernos estatal y nacional han invertido una parte substancial del erario en la creación de infraestructura, indispensable, para el desplazamiento, hospedaje y atención de los visitantes, así como en la promoción de ambos destinos vacacionales (García, Villerías y Tello, 2016).

En tanto, la modernización del sector secundario y primario no ha tenido lugar por la falta de inversiones públicas y privadas, a pesar de que la entidad cuenta con diversas áreas con características tanto físicas como socioeconómicas que podrían favorecer la creación de distintos proyectos productivos y, por lo tanto, atenuar la falta de alternativas laborales y los problemas sociales asociados con ella. Por ejemplo, a través del establecimiento de agroindustrias en los 
municipios de San Marcos, Florencio Villareal, Ayutla, San Luis Acatlán, Marquelia, Copala, Cuajinicuilapa, situados en la Costa Chica; o en Huamuxtitlán y Alpoyeca, en La Montaña (García, 2011).

Finalmente, los niveles de asimilación económica hallados en Guerrero dieron pauta para inferir que, en los próximos decenios, es probable que continúe la tercerización de los municipios de Acapulco, Zihuatanejo de Azueta y Chilpancingo. Asimismo, el comercio y los servicios podrían consolidarse como la principal fuente de trabajo de la PEA, especialmente en aquellas demarcaciones que albergan a las ciudades guerrerenses y en las jurisdicciones de la costa donde se ubicará el corredor turístico Acapulco-Zihuatanejo u otros proyectos de ese tipo. Por otro lado, en San Luis Acatlán, Metlatónoc, Tlacoapa, Atlamajalcingo del Monte, Malinaltepec, Acatepec y Zapotitlán Tablas podría diversificarse la estructura sectorial por el arribo inminente de compañías mineras, un acontecimiento que detonará la contaminación y destrucción del entorno físico de La Montaña y la porción serrana de la Costa Chica, así como distintos conflictos sociales debido a la expropiación de varios ejidos de esas regiones. 


\section{Bibliografía}

Aguilar, R. (2013). Niveles de asimilación económica del territorio en Baja California Sur. (Licenciatura en Geografía). Facultad de Filosofía y Letras, UNAM. México.

CONEVAL (2016). Rezago social a nivel zonas urbanas (AGEB urbanas). Recuperado de: http://www.coneval.gob.mx/Medicion/Paginas/Rezago_social_AGEB_2010.aspx

García, N. (2011). Los grados de asimilación económica del estado de Guerrero, a fines del siglo $X X$. (Doctorado en Geografía). Facultad de Filosofía y Letras, UNAM. México.

García, N., Villerías, S. y P. V. Tello (2015). Turismo y orientaciones sectoriales de los municipios costeros de Guerrero. En: Patrimonio, turismo y algo más... México. Juan Pablos Editor-Universidad Autónoma de Guerrero.

García, A. (1993). Asimilación económica del territorio (un nuevo enfoque en la interpretación regional del país). En Investigaciones Geográficas. Boletín núm. 27, 69-94. Recuperado de: http://www.scielo.org.mx/pdf/igeo/n27/n27a3.pdf

Gobierno del estado de Guerrero (2016). Municipios del estado de Guerrero. Gobierno del estado de Guerrero. Recuperado de: http://www.guerrero.gob.mx/?P=municipios

Hernández, R. (2007). Niveles de asimilación económica del Estado de México (Licenciatura en Geografía). Facultad de Filosofía y Letras, UNAM. México.

INEGI (2016). Sistema Estatal y Municipal de Base de Datos. Recuperado de: http://sc.inegi.org.mx/cobdem/

Mollinedo, G. (2008). Niveles de asimilación económica de Tamaulipas. (Licenciatura en Geografía). Facultad de Filosofía y Letras, UNAM. México.

Ortiz, M. I., Villaseñor, A. y L. Gerónimo (2009). El empleo de la Tipificación Probabilística en aspectos sociodemográficos y su aplicación en el ordenamiento territorial mediante el uso de SIGs. En: Espaciotiempo 4 Revista Latinoamericana de Ciencias Sociales y Humanidades, 76-91. Recuperado de: http://sociales.uaslp.mx/publicaciones/revistas

Privalovskaya, G. A. (1982). Regionalización del territorio de la URSS como método de estudio de la interacción entre la economía y el medio. En: Compilación temática del Instituto de 
Geografía de la URSS (traducción inédita). Archivo de traducciones del Instituto de Geografía Tropical. La Habana, Cuba.

Propin, E. y R. Thürmer (1986). Un nuevo enfoque metodológico de la regionalización económica: su aplicación en la República de Cuba. Wissenschaftliche Mitteilungen 18, 518. Institut für Geographie und Geoecologie. Leipzig.

Propin, E. y Á. Sánchez (1998). Niveles de asimilación económica del estado de Guerrero. Investigaciones Geográficas. Boletín núm. 37, 59-70. Recuperado de: http://www.scielo.org.mx/scielo.php?script=sci_arttext\&pid=S0188-46111998000300005

Unikel, L. (1978). El desarrollo urbano de México: Diagnóstico e implicaciones futuras. $2 d a$. Edición. México. El Colegio de México.

Villagrán, A. (2013). Niveles de asimilación económica del Estado de Tabasco (Licenciatura en Geografía). Facultad de Filosofía y Letras, UNAM. México. 